\title{
Multi-Channel Vibrotactile Display for Teleoperated Assembly
}

\author{
Thomas Debus, Tae-Jeong Jang*, Pierre Dupont, and Robert Howe
}

\begin{abstract}
This paper presents the design and testing of a multi-channel vibrotactile display. It is composed of a cylindrical handle with four embedded vibrating elements driven by piezoelectric beams. Vibrations are transmitted to the hands through arrays of pins. The device was tested in sensory substitution for conveying force information during a teleoperated peg insertion. Results show that the device is effective in reducing peak forces during the insertion task.
\end{abstract}

Keywords: Peg-in-hole insertion, teleoperation, vibration, vibrotactile display.

\section{INTRODUCTION}

Augmenting perception in man-machine systems consists of two parts. First, machine sensor data must be interpreted in a way appropriate to the task. Second, the task-specific information must be communicated to the operator in a format that addresses the limitations of human sensory information processing. This step involves tradeoffs on the number of bits to include in a sensory communication channel (e.g., how many possible force directions and magnitudes need be represented), the number of channels directed to a specific sense (e.g., how many different visual displays) and the number of senses to which information channels are directed (e.g., visual, auditory, tactile).

For a particular task, the utility of a sensory display is determined by how well it conveys task-specific information to the operator. On first glance, the utility of vibrotactile displays can appear modest. They have been shown to be inferior to force displays in terms of performances [5,7], and inferior to auditory displays in terms of communication channel width [7]. Furthermore, visual displays can be more natural due to the apparent dominance of vision over other senses [8].

Manuscript received January 16, 2004; revised July 13, 2004; accepted July 22, 2004. Recommended by Editor Keum-Shik Hong.

Thomas Debus and Pierre Dupont are with the Department of Aerospace and Mechanical Engineering, Boston University, 110 Cummington Street, Boston, MA 02215, USA (e-mails: \{tdebus, pierre\}@bu.edu).

Tae-Jeong Jang is with the Dept. of Electrical and Computer Engineering, Kangwon National University, 192-1 Hyoja2-dong, Chuncheon, Kangwon-do 200-701, KOREA (e-mail: jangtj@kangwon.ac.kr).

Robert Howe is with the Division of Engineering and Applied Sciences, Harvard University, 323 Pierce Hall, 29 Oxford Street, Cambridge, MA 02138, USA (e-mail: howe@deas.harvard.edu).

* Corresponding author.
Within the framework of human sensory processing, however, a large channel bandwidth requires more human processing to interpret and can, under certain conditions, actually increase task execution time [7]. In addition, the use of multiple visual displays that require the switching of attention can also slow task performance [1].

Additional factors can make the use of a display modality ineffective or costly. The operator's environment can contain noise sources that interfere with information transmission on a sensory channel. For example, during teleoperated surgery, vocal communication between non-operator team members can interfere with an auditory display. Similarly, overlaying visual displays (e.g., conveying force information) with an endoscope view during surgery can be costly and non-intuitive due to scene complexity and the wide range of viewpoints encountered.

To maximize effectiveness and minimize cost, a general design rule for sensory displays is to use the minimum possible information channel width on a sensory channel with low noise and which is not already receiving a signal requiring complex processing, i.e., avoid attention switching. Within this context, vibrotactile displays are promising for tasks that by default are rich in auditory and visual stimuli and for which force feedback is either imprecise (e.g., maintaining constant forces), too frail or costly (e.g., undersea oil operations), or is destabilizing (e.g., teleoperation with time delay).

This paper presents our first work on multi-channel vibrotactile displays for such applications. Much of the effort was focused on design issues. The next section presents a brief review of prior work on vibrotactile displays. The following section presents the mechanical and electrical designs of the multichannel vibrotactile device. The subsequent section describes an experimental evaluation of the tactile device during a peg-in-hole insertion task performed 
using a teleoperation system composed of a master PHANToM robot and a remote W.A.M robot. Conclusions are presented in the final section of the paper.

\section{BACKGROUND}

Force substitution via vibrotactile display is not a new concept. It has been an ongoing research topic for the past forty years. During this period, many vibrotactile systems have been designed, utilizing a broad range of actuators and spanning a wide range of applications, from undersea teleoperation 0 to aiding the blind 0 .

These devices can be roughly grouped according to the number of vibrating elements and, if more than one, whether the elements are widely separated or placed in a closely packed array. The latter case is exemplified by 0 in which a series of $0.1 \mathrm{~mm}$-diameter pins are arranged in a matrix configuration with six rows of 24 vibrating pins. Driven by piezoelectric crystals, the device is used to convert optical signals to vibrotactile information. Called Optacon ${ }^{\mathrm{TM}}$, it is used by blind people to feel the image viewed by an associated handheld camera.

In [10], a shape display consisting of a regular array of 10 pins actuated using SMA wire was used to convey tactile information. The device had a $-3 \mathrm{~dB}$ point of $40 \mathrm{~Hz}$ and detectable output that could still be felt at frequencies approaching $150 \mathrm{~Hz}$. The device was used to transmit information about the texture and contact state. The shape display was fast enough to keep up with finger speed when scanning over small object features; however, it was much more complex and expensive that traditional vibration displays that use piezoelectric crystals or small motors.

Devices exemplifying widely spaced vibrating elements include those described in 0 and [7]. In 0 , a system composed of three contact point actuators was designed. Each actuator used a disk-drive head positioning motor controlled via a digital angular position feedback controller to produce vibrations at a frequency range from DC to above $300 \mathrm{~Hz}$. The system was attached to the thumb, index, and middle finger and was used for a variety of tactual perceptual studies.

In [7], an experimental study of the use of auditory and vibrotactile display as sensory substitution for force feedback was conducted. Specifically, a vibrotactile display consisting of vibrating voice coils placed at the fingertips and palm of the dominant hand was used to perform a peg-in-hole task. Each vibrator had a resonant frequency of $250 \mathrm{~Hz}$, with intensity proportional to the magnitude of the associated force measured using force sensing resistors. Results showed that the vibrotactile or auditory displays did not speed up the task in the case of clear visual feedback. However, they did help in the cases of an obstructed view or time delay. Few vibrotactile devices are used in real industrial tasks. One notable exception can be found in 0 , where a vibrotactile system designed for the harsh condition of undersea hydraulic connector mating was presented. First a sensor using piezoelectric contact sensor was built and packed to fit into the manipulator gripper. Second, a simple voice coil motor mounted on an aluminum base was clamped on the unmodified master controller and was used as the vibrotactile display. The preliminary result showed that the device consistently eased the undersea connecting task.

Finally, some commercial systems have also been developed. One is The Cybertouch ${ }^{\mathrm{TM}}$ from Immersion. This device is composed of six vibrotactile displays attached to the palm and fingertips of the Cyberglove ${ }^{\circledR}$, a fully instrumented glove that provides up to 22 joint-angle measurements. Each actuator provides sustain vibrations at a frequency ranging form 0 to $125 \mathrm{~Hz}$, with a $1.2 \mathrm{~N}$ peak to peak amplitude at $125 \mathrm{~Hz}$. The system is used primary to interact with virtual environments.

\section{DISPLAY DESIGN}

The motivation behind the design was to create a vibrotactile device that could display complex signals during teleoperated assembly in a simple and efficient way to the user. To achieve this, a device that innovatively combined the advantage of multiple vibrating elements (i.e., richness of the display) with the advantage of closely packed array pins (i.e., large vibrating surfaces) was designed. An additional goal was to provide the most straightforward mapping between vibrations on the hand and coordinate directions on the master and remote manipulators. As a consequence, the decision was made to mount vibrotactile modules in the handle or stylus of the master device.

For ease of construction, it was decided to equally space four vibrotactile modules around the circumference of a cylindrical handle. As shown in Fig. 1, the four resonators can be used to represent

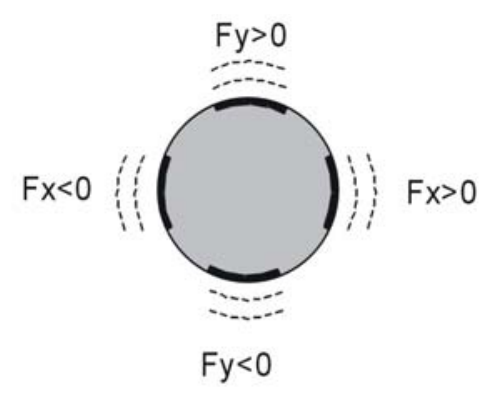

Fig. 1. View along cylinder axis showing vibration conditions of four resonators. 
positive and negative forces (or torques) with respect to two directions in the tool frame.

The vibrotactile modules were designed according to the following goals:

- They should not interfere with the natural grip of the handle.

- They should permit a variety of grasp locations on the handle.

- The vibration amplitude should be uniform over the surface of each module.

- The vibration amplitude should be independent of the grasp force.

These goals led to a design in which the vibrotactile modules were embedded flush with the cylindrical handle. Fig. 2 shows the device with one module disassembled. The module is about 1" by 2" allowing for a variety of grasps and finger sizes. To achieve a uniform level of vibration over the module's surface independent of grasp force, each module consists of a piezoelectric bimorph beam which sits freely in a cavity under 11 rows of 4 pins, spaced 0.1 inch apart. Vibrations are transmitted to the hand through the pins, which pass through holes in the module's cover. The

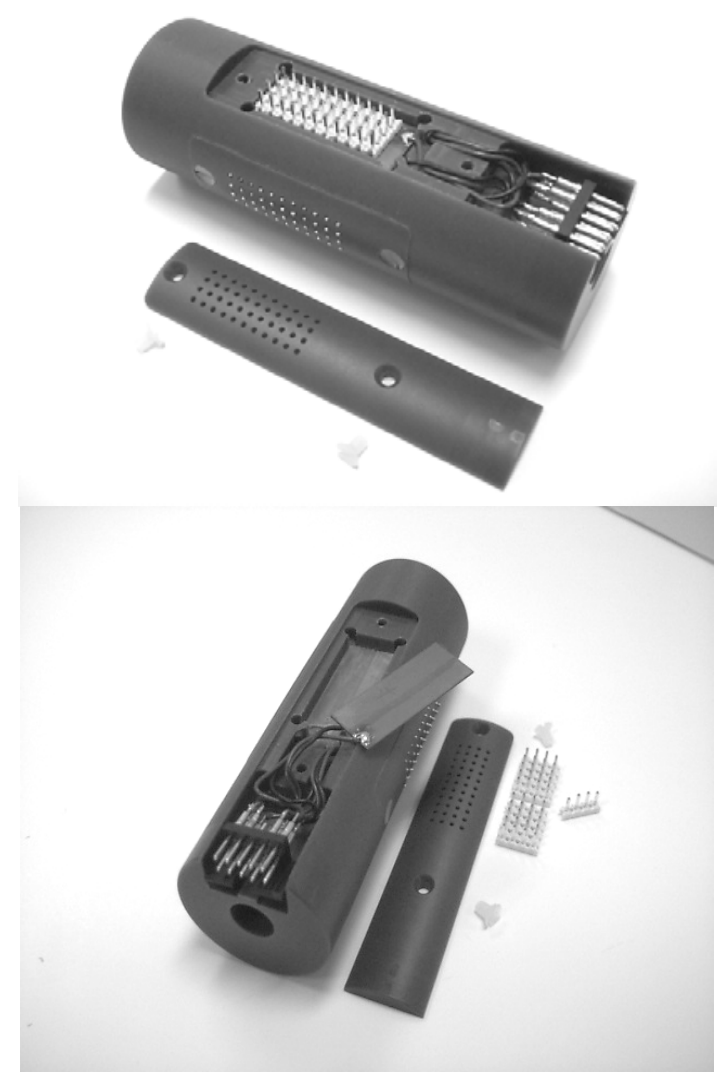

Fig. 2. Disassembled vibrotactile display revealing piezoelectric beam, cavity in which it vibrates and floating array of pins. pins in each row vary in length so that they conform to the radius of the module's cover plate.

By allowing free-free motion of the beam within the cavity, nodes of vibration (dead spots) are precluded such that the vibrotactile response is perceived to be uniform over the surface of the module. Furthermore, the depth of the cavity is such the beam can still vibrate freely under the pins regardless of grasp force. While a large grasp force does affect hand impedance and thus energy transfer from the pins to the hand, the beam and pins continue to vibrate against the skin.

The circuit driving the four modules is capable of delivering a variety of input voltages to the resonators (up to $\pm 80 \mathrm{~V} ; 50-600 \mathrm{~Hz}$; sinusoidal, triangular or square waves). The input voltage to each resonator is amplitude modulated at a frequency of $\sim 300 \mathrm{~Hz}$, which is the most sensitive frequency of human fingers 0 and is also the resonant frequency of the piezoelectric beams.

As currently designed, the two input channel voltages $(x, y)$ are mapped to four outputs by dividing them into their positive and negative parts $\left(x^{+}, x^{-}, y^{+}, y^{-}\right)$, each of which is used as the input to a resonator circuit. Assuming a force input, the output is as shown in Fig. 1.

The final device, as shown in Fig. 2, is a 4-inch long cylinder of outside diameter $11 / 4$ inch and inside diameter $3 / 8$ inch. The device has a total mass of 130 grams. While initially intended for mounting on the master handle, the final size of the device made it appropriate to hold in a power grasp (e.g., like holding a hammer). As a result the palm, thumbs, index and middle finger are in contact with one or more vibrotactile modules during this grasp. It was found in preliminary experiments that operators had more success gripping the master with a dextrous grasp (e.g., like holding a pen). When given the choice, operators preferred a direct and dextrous grasp of the master with the dominant hand while power grasping the vibrotactile device in the other hand.

\section{EXPERIMENTAL SETUP AND PROTOCOL}

It has been shown that with a clear real-time visual display, adding force information through sensory substitution does not speed up a task 0, [7]. For connector insertion, insertion time can measure the difficulty of the task; however, it does not give any indication about the quality of task performance. Consider, for example, an operator trying to 'force' his/her way through the socket while focused only on reducing time. Even if this operator successfully inserts the peg in record time, this strategy can still fail if the connector, socket or remote manipulator are damaged due to excessive force. The following experiments investigate how force information, 
conveyed through two sensory channels (vibration and visual), affects peak forces.

\subsection{Experimental setup}

The laboratory teleoperator testbed used in these experiments consists of a PHANToM haptic interface as the master controller and a Barrett Whole Arm Manipulator (WAM) as the remote robot (Fig. 3). The PHANToM (Model 1.5, Sensable Technologies, Cambridge, Mass., USA) is used as a passive 6 degree of freedom input device, and the motors are not activated. The WAM (Barrett Technologies, Cambridge, Mass., USA) is a redundant arm with 7 degrees of freedom. Only 5 axes are required for a peg-in-hole insertion task, and therefore the upper arm roll and final wrist roll axes are locked. Optical encoders measure the joint position on both robots, and velocities are computed using filtered backward differences. The workspace is roughly $0.2 \mathrm{~m}$ in diameter for the master robot and $1.0 \mathrm{~m}$ in diameter for the remote robot. The WAM robot is controlled by a dedicated RISC processor (Model DS1103, dSpace $\mathrm{GmbH}$, Paderborn, Germany) running at a $10 \mathrm{kHz}$ servo rate. The PHANToM joint data is read by a PC

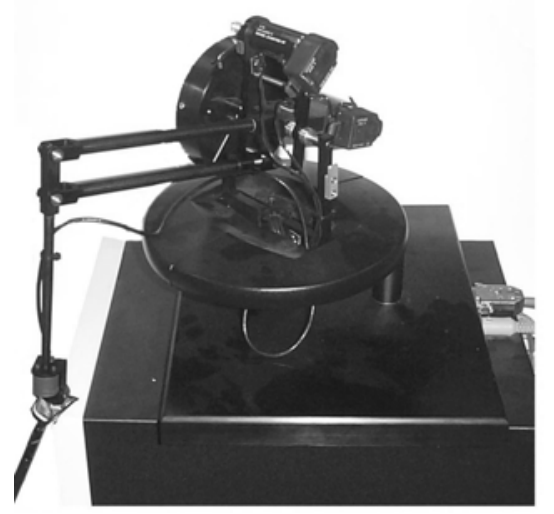

(a)

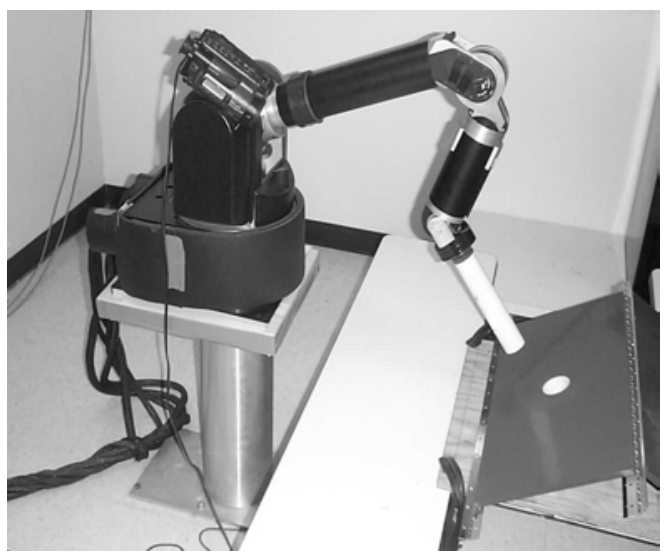

(b)

Fig. 3. (a) PHANToM master arm. (b) WAM remote robot arm with connector mating apparatus. at a rate of $1 \mathrm{kHz}$ and written into memory shared by the PC and the RISC processor every $200 \mathrm{~Hz}$. The block diagram of the experimental setup is depicted in Fig. 4.

Teleoperation is accomplished with a simple proportional-derivative controller with feedforward gravity and motor torque ripple compensation. Using either joint or Cartesian position, two control modes are available to the operator. First, an incremental joint-to-joint mapping between the PHANToM and the WAM can be used to roughly position and orient the peg. Second, an incremental Cartesian position mode, in which velocity and orientation of the master robot are mapped to the remote workspace, can be used to precisely insert the peg. In both cases, the master robot joints are converted to remote robot joint positions and velocity and then to torque commands by the following control laws.

$$
\tau_{i}^{\text {remote }}=K_{p_{i}}\left(q_{i}^{\text {master }}-q_{i}^{\text {remote }}\right)+K_{v_{i}}\left(\dot{q}_{i}^{\text {master }}-\dot{q}_{i}^{\text {remoto }}\right)+\tau_{i}^{\text {gravity }}+\tau_{i}^{\text {ripple }}
$$

Here $q_{i}, \dot{q}_{i}, \tau_{i}, \tau_{i}^{\text {gravity }}$ and $\tau_{i}^{\text {ripple }}$ are the $i^{\text {th }}$ components of joint position, velocity, torque, gravity compensation and motor torque ripple compensation. Experimentally, these gains were chosen to make the remote manipulator as stiff as possible in order to simulate typical industrial manipulators.

The connector-socket apparatus was simulated by a pair of PVC plastic tubes. The connector was 52.9 $\mathrm{mm}$ in diameter and $100 \mathrm{~mm}$ long. The socket had an inside diameter of $53 \mathrm{~mm}$ and was mounted perpendicularly to a planar surface that could be pivoted to a range of inclination angles between 0 and 57 degrees from the horizontal.

The operator can monitor the remote environment via three fixed video cameras. Fig. 5 shows the two close-up views and the distant view of the hole that are available to the operator. The two close-up views

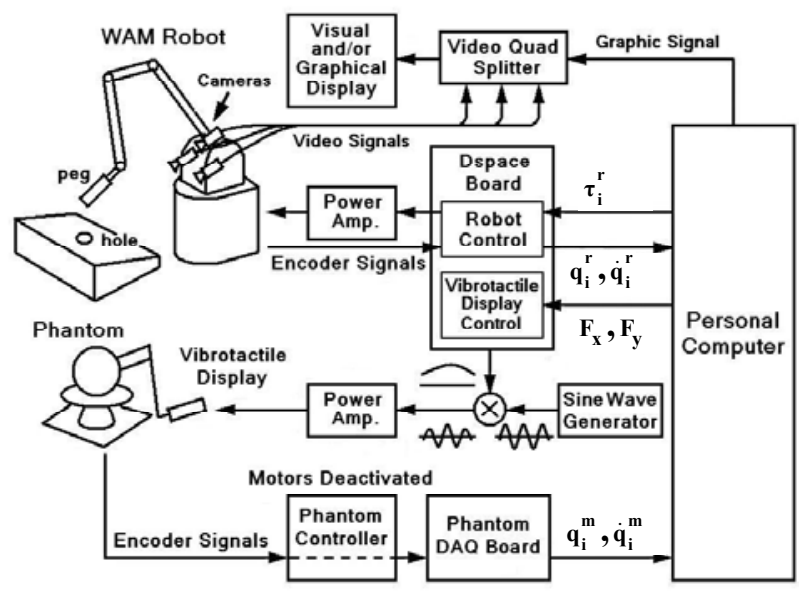

Fig. 4. Block diagram representation of the experimental setup. 
are angled to allow the user a stereoscopic view of the hole. The remaining slot of the quad display is used to show which control mode is active and other graphical information when needed (i.e., graphical display mode).

In addition to visual feedback, vibrotactile display and graphical representation of the force estimates are used to convey force information to the operator.

The vibrotactile device uses force estimates as inputs and then outputs vibrations proportional to the intensity of these forces. The cylindrical shape of the display makes it well designed for insertion tasks. In effect, the four resonators make a natural mapping to the sides of the hole, indicating to the user when and where the manipulated peg is in contact with the socket.

It is assumed that no force sensor is available; instead the compliance of the remote robot is used to estimate force. This technique only works in the quasi-static part of the insertion, when the operator is in contact with the environment. In the other phases of the insertion, the quasi-static assumption does not hold, and in this case, a simple dead zone is implemented to zero any transient force caused by the unmodeled dynamics of the system. As a result, any estimated forces below $20 \mathrm{~N}$ are ignored. This reduction of the force bandwidth does not reduce the performance of the device since forces are well above this threshold during the insertion phase.

To estimate forces, position errors between the master and the remote robot are computed and multiplied by a stiffness constant as shown in the following equation.

$$
F_{i}=k_{i}\left(x_{i}^{\text {master }}-x_{i}^{\text {remote }}\right)
$$

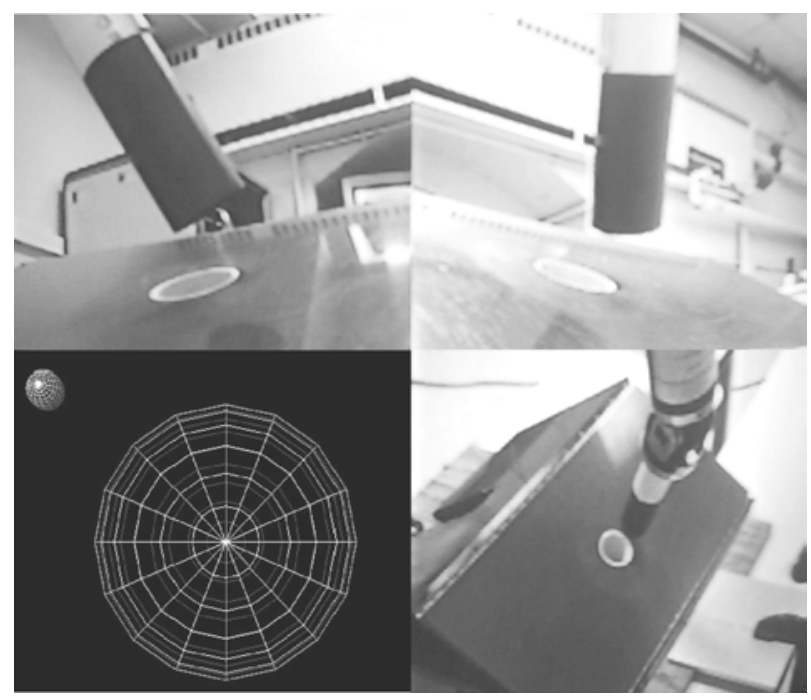

Fig. 5. Quad view used during the teleoperated insertion task.
Table 1. Remote manipulator stiffness gains.

\begin{tabular}{|c|c|c|c|}
\hline & X-direction & Y-direction & Z-direction \\
\hline $\mathrm{k}(\mathrm{N} / \mathrm{m})$ & $4000 \pm 50$ & $4800 \pm 50$ & $3500 \pm 50$ \\
\hline
\end{tabular}

The stiffness gains $k_{i}$ are directly related to the controller gains and the mechanical compliance of the remote manipulator. To identify the stiffness gains, calibrated masses have been placed on the remote manipulator end effector, and position displacements from the set points have been measured. Table 1 summarizes these results.

Looking at this table, one can see that the system is fairly compliant. For example, consider an insertion where the peak insertion force is around $100 \mathrm{~N}$. To produce this force a deflection of $25 \mathrm{~mm}$ is necessary. Such compliance facilitates the task; however, it also reduces the need for force substitution devices. This compliance was found to be mainly caused by tendon drive elasticity of the wrist. Finally, these force estimates are mapped to $\pm 10 \mathrm{~V}$ using a 24 bits $\mathrm{D} / \mathrm{A}$ converter. It is also important to note that.

The graphical display, as shown in Fig. 6, provides information similar to that of the vibrotactile device. The intensity and direction of the force estimates are mapped into a sphere of varying diameter and location. For example, if the peg is in contact with the bottom side of the hole, the sphere moves toward the bottom of the graphical representation of the hole. The distance from the center of the hole and the size of the sphere, are directly proportional to the force intensity. As with the vibrotactile display, if the force is below the $20 \mathrm{~N}$ threshold, no contact information is given.

Compared to the vibrotactile device, the graphical display provides richer information. In effect, there is no error in direction mapping as opposed to the vibrotactile device where amplitude modulation of four modules must indicate all possible force directions.

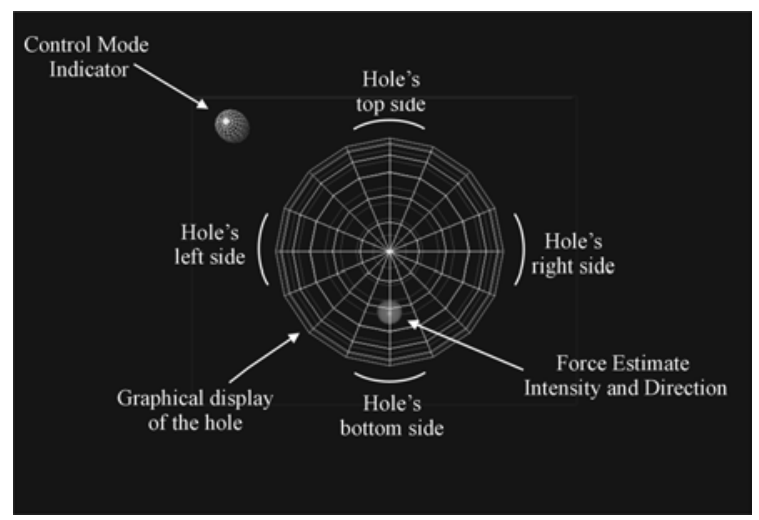

Fig. 6. The graphical display. 


\subsection{Experiment protocol}

Estimated peak force and insertion time are measured to assess task performance. Prior to data collection, the users are trained until they become fully accustomed to the system. Operator training for a particular display mode is terminated when the standard errors associated with their insertion time and peak force estimate plateau. Depending on the user, this can take between one and two hours.

Once fully trained, each operator completes two sets of experiments. For each set, three consecutive trials using each display are performed. A Latin square technique is used to determine the order in which the displays are tested. A total of nine subjects voluntarily participated in the experiment ( 7 males, 2 females, all with engineering backgrounds, ages 19-35). In the end, each subject performed a total of 18 insertions.

\section{EXPERIMENTAL RESULTS}

The results for the peg-in-hole insertion task are summarized in Table 2. Three feedback conditions were tested and compared: 1) visual feedback 2) graphical force display combined with visual feedback and, 3) vibrotactile force display combined with visual feedback. Mean peak force and insertion time were computed for each display. The table is separated into two groups of operators because three subjects were unable to decrease the peak force using either the graphical or the vibrotactile display. Although there is no statistical reason to discard data from these subjects, they are clearly not people one would hire for teleoperation involving anything expensive, fragile or dangerous.

Statistical analysis for the group composed of six subjects showed that visual information alone provided greater mean peak force than when combined with the graphical display $\left(\mu_{\text {visual }}^{\text {force }}=86.3>\mu_{\text {graph. }}^{\text {force }}=51.3, \mathrm{p}<0.001\right)$ or with the vibrotactile display $\left(\mu_{\text {visual }}^{\text {force }}=86.3>\mu_{\text {vib. }}^{\text {force }}=63.8, \mathrm{p}<0.011\right) \quad$.The graphical display, however, provided significantly lower mean peak force than the vibrotactile device $\left(\mu_{\text {graph. }}^{\text {force }}=51.3>\mu_{\text {vib. }}^{\text {force }}=63.8, p<0.051\right)$. In Fig. 7 , these results are

Table 2. Experimental results.

\begin{tabular}{|c|c|c|c|c|c|c|}
\hline \multirow{2}{*}{} & \multicolumn{3}{|c|}{$\begin{array}{c}\text { Mean Peak Force } \\
(\mathrm{N})\end{array}$} & \multicolumn{3}{c|}{$\begin{array}{c}\text { Mean Insertion Time } \\
(\mathrm{sec})\end{array}$} \\
\cline { 2 - 7 } & V.D & G.D & Vb.D & V.D & G.D & Vb.D \\
\hline $\begin{array}{c}9 \\
\text { subjects }\end{array}$ & 92.7 & 74.7 & 84.8 & 5.57 & 6.76 & 6.18 \\
\hline $\begin{array}{c}6 \\
\text { subjects }\end{array}$ & 86.3 & 51.3 & 63.8 & 5.28 & 6.92 & 6.55 \\
\hline
\end{tabular}

(V.D: visual display, G.D: graphical display, Vb.D: vibrotactile display) normalized using the visual display as a baseline. As shown, the peak force estimates are reduced by 40 percent with the graphical display and 26 percent with the vibrotactile display. When all 9 subjects are included, peak force reductions are still statistically significant; however, the percentage reduction is reduced to 20 percent and 9 percent for the graphical and vibrotactile displays, respectively.

Fig. 8 focuses on insertion time and shows that on average the insertion time is 31 percent and 24 percent longer using the graphical display and vibrotactile device, respectively. Detailed analysis showed that visual feedback alone provided a lower mean insertion time than when combined with the graphical display, $\left(\mu_{\text {visual }}^{\text {time }}=5.28<\mu_{\text {graph. }}^{\text {time }}=6.92, \mathrm{p}<0.001\right) \quad$ or $\quad$ the vibrotactile device $\left(\mu_{\text {visual }}^{\text {time }}=5.28<\mu_{\text {vib. }}^{\text {time }}=6.55, \mathrm{p}<0.056\right)$. However, the vibrotactile device provided lower mean insertion time than the graphical display $\left(\mu_{\text {vib. }}^{\text {time }}=6.55<\mu_{\text {graph. }}^{\text {time }}=6.92, \mathrm{p}<0.095\right)$.

\section{DISCUSSION}

These results demonstrate that basic force estimation together with sensory substitution can be effective in conveying useful task information during

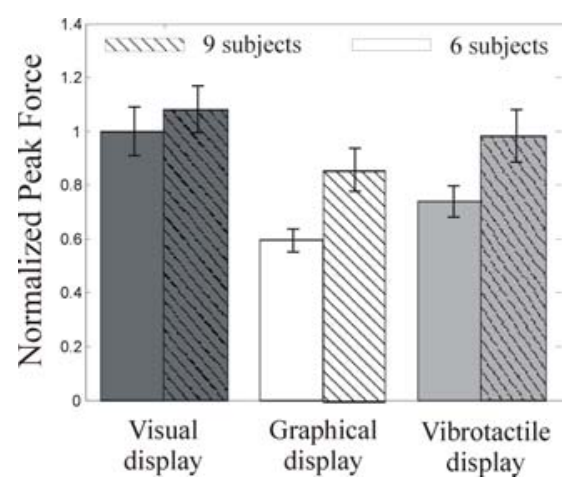

Fig. 7. Normalized peak force estimate as a function of display modes.

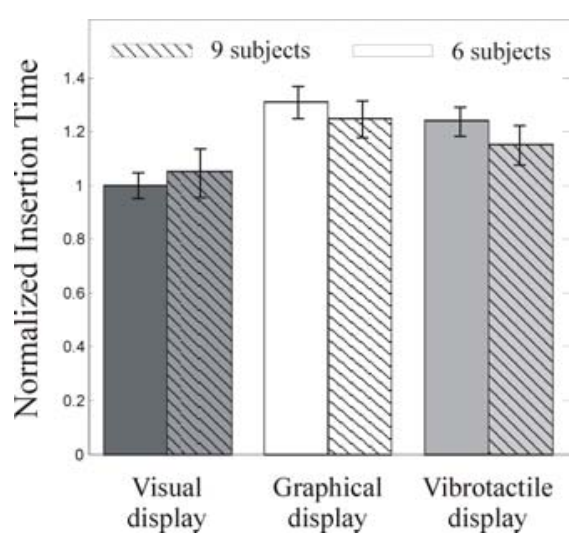

Fig. 8. Normalized insertion time as a function of display modes. 
teleoperation. In particular, both the graphical and vibrotactile displays led to a significant decrease in peak insertion force, and thus, reduced the risk of damage to the connector, socket and remote manipulator.

The superiority of the graphical display for force reduction is expected since mapping of force direction is transparent to the operator in comparison with the coarse directionality provided by the four-element vibrotactile device.

In agreement with prior results [7], use of the force displays in concert with a clear video signal increased insertion time. While the vibrotactile display seems to be more efficient than the graphical display, the level of significance is only 9.5 percent; more tests are needed to confirm this hypothesis.

A variety of factors can be used to explain the increased insertion time. First, as more information is made available to the operator, more cognitive processing is also required, inducing delay in the operator motor response. In this situation, the vibrotactile display presents an independent channel of communication that may facilitate the information processing.

A second factor is that torque, rather than force, provides better information for insertion tasks. While force helps by warning the user of misalignment; it does not directly indicate the corrective motion. Torque information is obviously more appropriate for indicating both misalignment and corrective action. Its effect on insertion time, however, is an open question.

Finally, robot compliance facilitates the insertion, reducing the need for graphical or vibrotactile display of force information. It is important to note that this compliance is manipulator and controller dependent. As a consequence a different manipulator could lead to different results. However, the trends observed in Figs. 7 and 8 agreed with the results presented in other papers, such as [7]. Nevertheless, more experiments are needed to investigate the relationship between manipulator compliance and task performances.

\section{CONCLUSIONS}

Although this is a preliminary study that requires further validation, these results indicate that the vibrotactile device provides a clear benefit in the intended application in terms of peak force reduction.

The main advantage of a vibrotactile device is to offer a cost effective, robust, and simple alternative to force feedback devices. In that respect, the proposed design presents a low cost and flexible device that can be used in a wider range of applications than previously designed vibrotactile displays. In teleoperated assembly the device can be used without interfering with normal task execution and without significant modifications to the existing manipulator.
Consequently, if the display fails; it has no impact on the manipulator's capabilities, and operations can continue without delay. In addition, avoiding the use of a visual display may prove advantageous for applications which already involve multiple or complex visual stimuli (e.g., surgery).

As for future work, a new vibrotactile display has been designed and built for use with a dextrous grasp. This cylindrical device weighs 40 grams, has a 0.6 " diameter and 4.75 " length. Three vibrotactile arrays around its perimeter are mounted to contact the fingertips when the device is gripped like a pen. The effect of mounting this device as the master manipulator handle will be studied along with a comparison of sensory substitution using complex signals such as torque information.

\section{REFERENCES}

[1] A. Bejczy, R. Dotson, J. Brown, and J. Lewis, "Manual control of manipulator forces and torques using graphic display," Proc. of the IEEE International Conference on Cybernetics and Society, Seattle, Washington, pp. 691-698, October 1981.

[2] J. Bliss, M. Katcher, C. Rogers, and R. Shepard, "Optical-to-tactile image conversion for the blind," IEEE Trans. on Man-Machine Systems, vol. 11, no. 1, pp. 58-65, March 1970.

[3] K. Boff and J. Lincoln, Engineering Data Compendium: Human Perception and Performance, Ohio: H. G. Anderson Aerospace Medical Research Laboratory, 1988.

[4] T. Debus, J. Stoll, R. Howe, and P. Dupont, "Cooperative human and machine perception in teleoperated assembly," Experimental Robotics VII, D. Rus and S. Singh (Eds.), Springer-Verlag, New York, pp. 51-60, 2001.

[5] T. Debus, T. Becker, P. Dupont, T.-J. Jang, and R. Howe, "Multichannel vibrotactile display for sensory substitution during teleoperation," Proc. of the SPIE International Symposium on Intelligent Systems and Advanced Manufacturing, Newton, MA, pp. 42-49, October 2001.

[6] J. Dennerlein, P. Millman, and R. Howe, "Vibrotactile feedback for industrial telemanipulators," Proc. of the Sixth Annual Symposium on Haptic Interfaces for Virtual Environment and Teleoperator Systems, ASME International Mechanical Engineering Congress and Exposition, vol. 61, Dallas, pp. 189-195, November 1997.

[7] M. Massimino, Sensory Substitution for Force Feedback in Space Teleoperation, MIT Ph.D. Thesis, Department of Mechanical Engineering, MIT, June 1992.

[8] M. Posner, M. Nissen, and R. Klein, "Visual dominance: an information-processing account 
of its origins and significance," Psychological Review, vol. 82, no. 2, pp. 157-171, 1976.

[9] H. Tan and W. Rabinowitz, "A new multi-finger tactual display," Proc. of the ASME Dynamic Systems and Control Division, Atlanta, pp. 515$522,1996$.

[10] P. Wellman, W. Peine, G. Favalora, and R. Howe, "Mechanical design and control of a highbandwidth shape memory alloy tactile display," Experimental Robotics V, A. Casals and A. T. de Almeida (Eds.), Lecture notes in control and information sciences, vol. 232, Springer-Verlag, pp. 56-66, 1998.

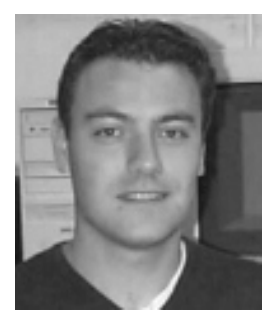

Thomas Debus received a B.S. degree in mechanical engineering from the University of Versailles, France, in 1995, and an M.S. degree in mechanical engineering in 2000 from Boston University, Boston, USA, where he is currently pursuing a Ph.D. degree. His research interests include the planning and analysis of robotics tasks in unstructured environments, teleoperated systems and man-machine cooperation.

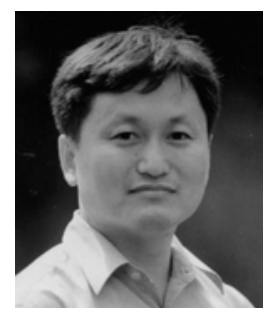

Tae-Jeong Jang received the B.S., M.S. and Ph.D. degrees in Control and Intrumentation Engineering from Seoul National University, Korea, in 1986, 1988 and 1994, respectively. He joined Kangwon National University in 1995 where he is currently an Associate Professor in the Department of Electrical and Computer Engineering. His research interests include haptic interfaces, vibrotactile displays, control theory and its applications, and digital communications.

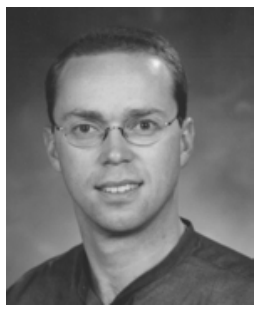

Pierre Dupont received the B.S., M.S. and Ph.D. degrees in Mechanical Engineering from Rensselaer Polytechnic Institute in 1982, 1984 and 1988, respectively. He was a postdoctoral fellow with the Center for Intelligent Control Systems at Harvard University from 1988 to 1990 . He joined Boston University in 1990 where he is currently an Associate Professor in the Department of Aerospace and Mechanical Engineering. His research interests include robotics, control systems and medical applications. He is an Associate Editor of the IEEE Transactions on Robotics, a senior member of the IEEE and a member of the ASME.

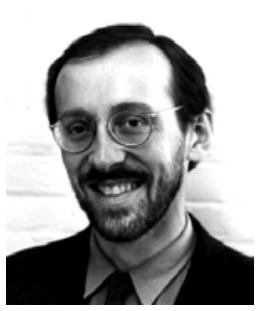

Robert D. Howe received a B.A. in physics from Reed College, then worked as a design engineer in the electronics industry. He attended graduate school at Stanford University and received a Ph.D. in mechanical engineering in 1990 . He then joined the faculty of the Division of Engineering and Applied Sciences at Harvard University, where he is currently Gordon McKay Professor of Engineering. Dr. Howe received the National Science Foundation Young Investigator Award in 1993 and the Whitaker Foundation Biomedical Engineering Research Grant (career development award) in 1995.

Dr. Howe's research interests focus on robot and human hands and the sense of touch. He has developed new tactile sensors for robot fingers, and demonstrated novel control algorithms based on these tactile sensor signals. His biomechanics research projects involve measurement of the mechanical impedance of human hands and modeling of mechanoreceptor neural function. Biomedical applications of this work include the development of new technology for minimally invasive surgery. 